\begin{tabular}{|c|l|}
\hline Title & Scattering theory for the Hartree equation \\
\hline Author(s) & Hayashi, N.; Naumkin, P.I; Ozawa, T. \\
\hline Citation & Hokkaido University Preprint Series in Mathematics, 358, 1-14 \\
\hline Issue Date & 1996-11-1 \\
\hline DOI & 10.14943/83504 \\
\hline Doc URL & http://hdl.handle.net/2115/69108 \\
\hline Type & bulletin (article) \\
\hline File Information & pre358.pdf \\
\hline
\end{tabular}

Instructions for use 


\section{SCATTERING THEORY FOR THE HARTREE EQUATION}

N. Hayashi, P.I. Naumkin and T. Ozawa

Series \#358. November 1996 


\section{HOKKAIDO UNIVERSITY PREPRINT SERIES IN MATHEMATICS}

\#334 B. Khanedani and T. Suwa, First variation of horomorphic forms and some applications, 11 pages. 1996.

\#335 J. Seade and T. Suwa, Residues and topological invariants of singular holomorphic foliations ${ }^{1}, 28$ pages. 1996.

\#336 Y. Giga, M.E. Gurtin and J. Matias, On the dynamics of crystalline motions, 67 pages. 1996.

\#337 I. Tsuda, A new type of self-organization associated with chaotic dynamics in neural networks, 22 pages. 1996.

\#338 F. Hiroshima, A scaling limit of a Hamiltonian of many nonrelativistic particles interacting with a quantized radiation field, 34 pages. 1996.

\#339 N. Tominaga, Analysis of a family of strongly commuting self-adjoint operators with applications to perturbed Dirac operators, 29 pages. 1996.

\#340 A. Inoue, Abel-Tauber theorems for Fourier-Stieltjes coefficients, 17 pages. 1996.

\#341 G. Ishikawa, Topological classification of the tangent developables of space curves, 19 pages. 1996.

\#342 Y. Shimizu, A remark on estimates of bilinear forms of gradients in Hardy space, 8 pages. 1996.

\#343 N. kawazumi and S. Morita, The primary approximation to the cohomology of the moduli space of curves and cocycles for the stable characteristic classes, 11 pages. 1996.

\#344 M.-H. Giga and Y. Giga, A subdifferential interpretation of crystalline motion under nonuniform driving force, 18 pages. 1996.

\#345 A. Douai and H. Terao, The determinant of a hypergeometric period matrix, 20 pages. 1996.

\#346 H. Kubo and K. Kubota, Asymptotic behaviors of radially symmetric solutions of $\square u=|u|^{p}$ for super critical values $p$ in even space dimensions, 66 pages. 1996.

\#347 T. Nakazi and T. Yamamoto, Weighted Norm Inequalities For Some Singular Integral Operators, 17 pages. 1996.

\#348 Y. Ito and I. Nakamura, Hilbert schemes and simple singularities $A_{n}$ and $D_{n}, 22$ pages. 1996

\#349 R. Agemi and K. Yokoyama, The null condition and global existence of solutions to systems of wave equations with different speeds, 42 pages. 1996

\#350 F. Hiroshima, Weak coupling limit with a removal of an ultraviolet cut-off for a Hamiltonian of particles interacting with a massive scalar field, 21 pages. 1996.

\#351 T. Nakazi and Y. Watatani, Invariant subspace theorems for subdiagonal algebras, 22 pages. 1996.

\#352 Y. Nishiura and H. Suzuki, Nonexistence of stable turing patterns with smooth limiting interfacial configurations in higher dimensional spaces, 21 pages. 1996.

\#353 Y.-G. Chen, Y. Giga and K. Sato, On instant extinction for very fast diffusion equations, 9 pages. 1996.

\#354 A. Gyoja and H. Yamashita, Associated variety, Kostant-Sekiguchi correspondence, and locally free $U(\mathrm{n})$-action on Harish-Chandra modules, 25 pages. 1996.

\#355 G. Ishikawa, Topology of plane trigonometric curves and the strangeness of plane curves derived from real pseudo-line arrangements, 18 pages. 1996.

\#356 N.H. Bingham and A. Inoue, The Drasin-Shea-Jordan theorem for Hankel transforms of arbitrarily large order, 13 pages. 1996.

\#357 S. Izumiya, Singularities of solutions for first order partial differential equations, 20 pages. 1996. 


\title{
SCATTERING THEORY FOR THE HARTREE EQUATION
}

\author{
NaKaO HaYashi ${ }^{1}$, Pavel I. NaUmKIn ${ }^{2}$ and Tohru OzaWa ${ }^{3}$ \\ ${ }^{1}$ Department of Applied Mathematics, Science University of Tokyo \\ 1-3, Kagurazaka, Shinjuku-ku, Tokyo 162, JAPAN \\ e-mail : nhayashi@rs.kagu.sut.ac.jp \\ ${ }^{2}$ Instituto de Fisica y Matematicas, Universidad Michoacana \\ AP 2-82, CP 58040, Morelia, Michoacana, MEXICO \\ e-mail : naumkin@ifm1.ifm.umich.mx \\ and \\ ${ }^{3}$ Department of Mathematics, Hokkaido University \\ Sapporo 060, JAPAN
}

Abstract. We study the scattering problem for the Hartree equation

$$
\left\{\begin{array}{l}
i \partial_{t} u=-(1 / 2) \Delta u+f\left(|u|^{2}\right) u, \quad(t, x) \in \mathbf{R} \times \mathbf{R}^{n}, \\
u(0, x)=u_{0}(x), \quad x \in \mathbf{R}^{n}
\end{array}\right.
$$

where

$$
f\left(|u|^{2}\right)=V *|u|^{2}, \quad V(x)=\lambda|x|^{-1}, \lambda \in \mathbf{R}, \quad n \geq 2 .
$$

We prove that for any $u_{0} \in H^{0, \gamma} \cap H^{\gamma, 0}$, with $\frac{1}{2}<\gamma<\frac{n}{2}$, such that the value $\epsilon=\left\|u_{0}\right\|_{0, \gamma}+\left\|u_{0}\right\|_{\gamma, 0}$ is sufficiently small, there exists unique $u_{ \pm} \in H^{\sigma, 0} \cap H^{0, \sigma}$ with $\frac{1}{2}<\sigma<\gamma$, such that for all $|t| \geq 1$

$$
\left\|u(t)-\exp \left(\mp i f\left(\left|\hat{u}_{ \pm}\right|^{2}\right)\left(\frac{x}{t}\right) \log |t|\right) U(t) u_{ \pm}\right\|_{L^{2}} \leq C \epsilon|t|^{-\mu+C \epsilon},
$$

where $\mu=\min \left(1, \frac{\gamma}{2}\right), \hat{\phi}$ denotes the Fourier transform of $\phi$ defined by

$$
\hat{\phi}(x)=\frac{1}{(2 \pi)^{n / 2}} \int e^{-i x \xi} \phi(\xi) d \xi,
$$

$U(t)$ is the free Schrödinger evolution group and $H^{m, s}$ is the weighted Sobolev space defined by

$$
H^{m, s}=\left\{\phi \in \mathcal{S}^{\prime} ;\|\phi\|_{m, s}=\left\|\left(1+|x|^{2}\right)^{s / 2}(1-\Delta)^{m / 2} \phi\right\|_{L^{2}}<\infty\right\} .
$$

AMS Classification 35Q55 
$\S 1$ Introduction. This paper is devoted to the study of the asymptotic behavior for large time of solutions to the Cauchy problem for the Hartree equation

$$
\left\{\begin{array}{l}
i \partial_{t} u=-(1 / 2) \Delta u+f\left(|u|^{2}\right) u, \quad(t, x) \in \mathbf{R} \times \mathbf{R}^{n} \\
u(0, x)=u_{0}(x), \quad x \in \mathbf{R}^{n}
\end{array}\right.
$$

where

$$
f\left(|u|^{2}\right)=V *|u|^{2}=\int V(x-y)|u|^{2}(y) d y, V(x)=\lambda|x|^{-1}, \lambda \in \mathbf{R} \quad \text { and } \quad n \geq 2 .
$$

There is a large amount of literature on the Cauchy problem for (1.1) and the asymptotic behavior in time of solutions for (1.1) with $\lambda>0$ [2,3,6-13]. The scattering problem for (1.1) has only been studied in the framework of the nonexistence of scattering states $[11,12]$ or of the existence of modified wave operators [5]. It seems that the decay rates of solutions to (1.1) obtained in [3,7-10] through the pseudoconformal conservation law are not sufficient to obtain the existence and uniquness of the modified scattering states (see [14] for a study in this direction ). The only exception is [9], where the existence of solutions with the same decay rate as in the free case is proved and the asymptotic profile of the solutions is studied. In the present paper we propose a new setting for the study of large time behavior of solutions to (1.1) to make clear the connection with the theory of long range scattering for (1.1). Although the method of the paper follows [9], the argument here is different in some respects from the previous one and requires a number of sharp estimates.

We denote by $\mathcal{F} \phi$ or $\hat{\phi}$ the Fourier transformation of $\phi$ defined by

$$
\mathcal{F} \phi(\xi)=\frac{1}{(2 \pi)^{\frac{n}{2}}} \int e^{-i x \xi} \phi(x) d x
$$

and let $\mathcal{F}^{-1} \phi(x)$ be the inverse Fourier transformation of $\phi$ :

$$
\mathcal{F}^{-1} \phi(x)=\frac{1}{(2 \pi)^{\frac{n}{2}}} \int e^{i x \xi} \phi(\xi) d \xi .
$$

The free Schrödinger evolution group $U(t)=e^{\frac{i t}{2} \Delta}$ is given by

$$
U(t) \phi=\frac{1}{(2 \pi i t)^{\frac{n}{2}}} \int e^{\frac{i|x-y|^{2}}{2 t}} \phi(y) d y=\mathcal{F}^{-1} e^{-\frac{i t}{2}|\xi|^{2}} \mathcal{F} \phi .
$$

We define the weighted Sobolev space $H^{m, s}$ by $H^{m, s}=\left\{\phi \in \mathcal{S}^{\prime} ;\|\phi\|_{m, s}=\|(1+\right.$ $\left.\left.|x|^{2}\right)^{s / 2}(1-\Delta)^{m / 2} \phi \|<\infty\right\}$, where $m, s \in \mathbf{R}$ and $\|\cdot\|$ denotes the usual $L^{2}$ norm.

We now state our results in this paper. 
Theorem 1.1. We assume that $u_{0} \in H^{\gamma, 0} \cap H^{0, \gamma}$ and $\left\|u_{0}\right\|_{\gamma, 0}+\left\|u_{0}\right\|_{0, \gamma}=\epsilon$, where $\epsilon$ is sufficiently small and $\frac{1}{2}<\gamma<\frac{n}{2}, n \geq 2$.

Then there exists a unique.global solution $u$ of the Hartree equation (1.1) such that $u \in C\left(\mathbf{R} ; H^{\gamma, 0} \cap H^{0, \gamma}\right)$ and

$$
\sup _{\alpha \in\left[\frac{1}{2}, \sigma\right]} \sup _{t \in \mathbf{R}}(1+|t|)^{-\alpha}\|u(t)\|_{p(\alpha)} \leq C \epsilon
$$

where $\frac{1}{2}<\sigma<\gamma, p(\alpha)=\frac{2 n}{n-2 \alpha}$.

Theorem 1.2. Let $u$ be the solution of (1.1) obtained in Theorem 1.1.

Then for any $u_{0}$ satisfying the condition of Theorem 1.1, there exist unique functions $u_{ \pm} \in H^{\sigma, 0} \cap H^{0, \sigma}, \frac{1}{2}<\sigma<\gamma$, such that for all $|t| \geq 1$

$$
\left\|u(t)-\exp \left(\mp i f\left(\left|\hat{u}_{ \pm}\right|^{2}\right)\left(\frac{x}{t}\right) \log |t|\right) U(t) u_{ \pm}\right\| \leq C \epsilon|t|^{-\mu+C \epsilon}
$$

where $\mu=\min \left(1, \frac{\gamma}{2}\right)$.

In the previous paper [9] the following results were shown.

Proposition 1.1. We assume that $u_{0} \in H^{\gamma, 0} \cap H^{0, \gamma}$ and $\left\|u_{0}\right\|_{\gamma, 0}+\left\|u_{0}\right\|_{0, \gamma}=\epsilon$, where $\epsilon$ is sufficiently small and $\gamma>n / 2$.

Then there exists a unique global solution $u$ of the Hartree equation (1.1) such that $u \in C\left(\mathbf{R} ; H^{\gamma, 0} \cap H^{0, \gamma}\right)$ and

$$
\|u(t)\|_{\infty} \leq C \epsilon(1+|t|)^{-n / 2} .
$$

Proposition 1.2. Let $u$ be the solution of (1.1) stated in Proposition 1.1.

Then for any $u_{0} \in H^{\gamma, 0} \cap H^{0, \gamma}$ there exist unique functions $\Phi \in L^{\infty}$ and $\hat{u}_{+} \in$ $L^{\infty} \cap L^{2}$ such that

$$
\left\|\int_{1}^{t} f\left(|\hat{u}(\tau)|^{2}\right) \frac{d \tau}{\tau}-f\left(\left|\hat{u}_{+}\right|^{2}\right) \log t-\Phi\right\|_{\infty} \leq C \epsilon t^{-\xi \eta} \quad \text { for } \quad t \geq 1
$$

and

$$
\left\|\mathcal{F}(U(-t) u)(t) \exp \left(i \int_{1}^{t} f\left(|\hat{u}(\tau)|^{2}\right) \frac{d \tau}{\tau}\right)-\hat{u}_{+} e^{i \Phi}\right\|_{k} \leq C \epsilon t^{-\xi} \quad \text { for } \quad t \geq 1
$$

where $k=2$ or $\infty, 0<\eta<2 / n, n / 2+2 \xi<\gamma$, and $0<\xi<1$. Furthermore we have the asymptotic formula for large time $t$ uniformly with respect to $x \in \mathbf{R}^{n}$

$$
u(t, x)=\frac{1}{(2 \pi i t)^{\frac{n}{2}}} \hat{u}_{+}\left(\frac{x}{t}\right) \exp \left(i \frac{|x|^{2}}{2 t}-i f\left(\left|\hat{u}_{+}\right|^{2}\right)\left(\frac{x}{t}\right) \log t\right)+O\left(\epsilon t^{-\frac{n}{2}-\xi \eta}\right)
$$


and the estimate

$$
\left\|\mathcal{F}(U(-t) u)(t)-\hat{u}_{+} \exp \left(-i f\left(\left|\hat{u}_{+}\right|^{2}\right) \log t\right)\right\|_{k} \leq C \epsilon t^{-\xi \eta}
$$

where $k=2$ or $\infty$.

If we write the formula (1.3) in the form

$$
u(t, x)=\exp \left(-i f\left(\left|\hat{u}_{+}\right|^{2}\right)\left(\frac{x}{t}\right) \log t\right) U(t) u_{+}+O\left(\epsilon t^{-\frac{n}{2}-\xi \eta}\right)
$$

then we get the inequality

$$
\left\|u(t)-\exp \left(-i f\left(\left|\hat{u}_{+}\right|^{2}\right)\left(\frac{x}{t}\right) \log t\right) U(t) u_{+}\right\|_{\infty} \leq C \epsilon t^{-\frac{n}{2}-\xi \eta}
$$

which is similar to (1.2) but gives the estimate in the uniform norm. The setting in Theorem 1.2 fits more closely to the theory of long-range scattering developed in [5], where the existence of modified wave operators for (1.1) has been proved. Roughly speaking the result of Theorem 1.2 implies the asymptotic completeness of the modified wave operators. Another advantage of the setting in Theorem 1.2 over the previous one in Proposition 1.2 is that for the $L^{2}$ theory of scattering one needs only the requirement $\gamma>1 / 2$ for the index of the weighted Sobolev spaces as compared to the previous assumption, namely $\gamma>n / 2$.

We organize our paper as follows. Below we explain the necessary notations and then in Section 2 we give some preliminary results. In Lemma 2.1 we formulate wellknown embedding results for the Sobolev spaces. Lemma 2.2 gives the sharp time decay estimate of $L^{p}$ norm of the function in term of the free evolution group $U(t)$. And in Lemma 2.3 we prove the estimates of the nonlinearity of equation (1.1) in the weighted Sobolev spaces. In Section 3 we prove Theorems 1.1-1.2 by using a priori estimates of the solutions obtained in Lemmas $3.1-3.3$ in a space $X_{T}$. The function space $X_{T}$ is the following

$$
\begin{aligned}
& X_{T}=\left\{\phi \in C\left([-T, T] ; \mathcal{S}^{\prime}\right) ;\|\phi \mid\|_{X_{T}}=\sup _{t \in[-T, T]}(1+|t|)^{-C \epsilon}\|\phi(t)\|_{\gamma, 0}\right. \\
& \left.\quad+\sup _{t \in[-T, T]}(1+|t|)^{-C \epsilon}\|U(-t) \phi(t)\|_{0, \gamma}+\sup _{\alpha \in\left[\frac{1}{2}, \sigma\right]} \sup _{t \in[-T, T]}(1+|t|)^{-\alpha}\|\phi(t)\|_{p(\alpha)}<\infty\right\}
\end{aligned}
$$

where $p(\alpha)=\frac{2 n}{n-2 \alpha}, \frac{1}{2}<\sigma<\gamma<\frac{n}{2}$.

We consider below the case $t>0$ only since the opposite case is treated analogously.

Notation and function spaces. We let $\partial_{j}=\partial / \partial x_{j}, \partial^{l}=\partial_{1}^{l_{1}} \cdots \partial_{n}^{l_{n}}, l \in(\mathbf{N} \cup$ $\{0\})^{n}, M=M(t)=\exp \left(i|x|^{2} / 2 t\right), J_{j}=J_{j}(t)=\left(x_{j}+i t \partial_{j}\right)=U(t) x_{j} U(-t)$, 
$J=\left(J_{1}, \cdots, J_{n}\right)=U(t) x U(-t)$, and $|J|^{\zeta}=U(t)|x|^{\zeta} U(-t), \zeta \in[0, \infty)$. We introduce some function spaces. $L^{p}=\left\{\phi \in \mathcal{S}^{\prime} ;\|\phi\|_{p}<\infty\right\}$, where $\|\phi\|_{p}=\left(\int|\phi(x)|^{p} d x\right)^{1 / p}$ if $1 \leq p<\infty$ and $\|\phi\|_{\infty}=\operatorname{ess.sup}\left\{|\phi(x)| ; x \in \mathbf{R}^{n}\right\}$ if $p=\infty$. For simplicity we let $\|\phi\|=\|\phi\|_{2}$. Weighted Sobolev space $H_{p}^{m, s}$ defined by $H_{p}^{m, s}=\left\{\phi \in \mathcal{S}^{\prime} ;\|\phi\|_{m, s, p}=\right.$ $\left.\left\|\left(1+|x|^{2}\right)^{s / 2}(1-\Delta)^{m / 2} \phi\right\|_{p}<\infty\right\}, m, s \in \mathbf{R}, 1 \leq p \leq \infty ; H^{m, s}=H_{2}^{m, s},\|\cdot\|_{m, s}=$ $\|\cdot\|_{m, s, 2}$. We let $(\psi, \phi)=\int \psi \cdot \bar{\phi} d x$. Denote by $\dot{B}_{p, q}^{s}$ the homogeneous Besov space with the semi-norm

$$
\|\psi\|_{\dot{B}_{p, q}^{s}}=\left(\int_{0}^{\infty} y^{-1-\xi q} \sup _{|z| \leq y} \sum_{|k| \leq[s]}\left\|\partial^{k}\left(\psi_{(z)}-\psi\right)\right\|_{p}^{q} d y\right)^{1 / q},
$$

where $s=[s]+\xi, 0<\xi<1, \psi_{(z)}(x)=\psi(x+z)$ and $[s]$ is the largest integer less than $s$. We let $C(I ; E)$ be the space of continuous functions from an interval $I$ to a Banach space $E$. Different positive constants might be denoted by the same letter $C$. If necessary, by $C(*, \cdots, *)$ we denote constants depending on the quantities appearing in parentheses.

We note that $U(t)=M(t) D(t) \mathcal{F} M(t)$, where $D(t)$ is the dilation operator defined by $(D(t) \psi)(x)=\frac{1}{(i t)^{\frac{n}{2}}} \psi\left(\frac{x}{t}\right)$ and

$$
U(-t)=M(-t) \mathcal{F}^{-1} D(t)^{-1} M(-t)=M(-t) i^{n} \mathcal{F}^{-1} D\left(\frac{1}{t}\right) M(-t),
$$

since $D(t)^{-1}=i^{n} D\left(\frac{1}{t}\right)$. By using the above identities we easily see that

$$
\begin{aligned}
J_{j}(t) & =U(t) x_{j} U(-t)=M(t) D(t) \mathcal{F} M(t) x_{j} M(-t) \mathcal{F}^{-1} i^{n} D\left(\frac{1}{t}\right) M(-t) \\
& =M(t) D(t) i^{n}\left(i \partial_{j}\right) D\left(\frac{1}{t}\right) M(-t)=M(t) D(t) i^{n} D\left(\frac{1}{t}\right)\left(i t \partial_{j}\right) M(-t) \\
& =M(t)\left(i t \partial_{j}\right) M(-t)
\end{aligned}
$$

and $|J|^{\zeta}(t)=M(t)\left(-t^{2} \Delta\right)^{\frac{\zeta}{2}} M(-t), \quad \zeta \in[0, \infty)$.

\section{$\S 2$ Preliminaries.}

Lemma 2.1. Let $q, r$ be any numbers satisfying $1 \leq q, r \leq \infty$, and let $j, m$ be any real numbers satisfying $0 \leq j<m$. If $u \in H_{r}^{m, 0} \cap L^{q}$, then

$$
\left\|(-\Delta)^{j / 2} u\right\|_{p} \leq C\left\|(-\Delta)^{m / 2} u\right\|_{r}^{a}\|u\|_{q}^{1-a}
$$

where $(1 / p)=(j / n)+a((1 / r)-(m / n))+(1-a) / q$ for all $a$ in the interval $(j / m) \leq$ $a \leq 1$, where $C$ is a constant depending only on $n, m, j, q, r, a$, with the following exception: if $m-j-(n / r)$ is a nonnegative integer, then (2.1) holds for any $(j / m) \leq$ $a<1$.

For Lemma 2.1 see, e.g., [4]. 
Lemma 2.2. We let $u(t, x)$ be a smooth function. Then we have

$$
\|u(t)\|_{p(\alpha)} \leq C|t|^{-\alpha}\|\mathcal{F} U(-t) u(t)\|_{p(\alpha)}+C|t|^{-\alpha-\nu}\|U(-t) u(t)\|_{0, \gamma} \quad \text { for } \quad|t| \geq 1
$$

where $p(\alpha)=\frac{2 n}{n-2 \alpha}, \alpha \in\left[\frac{1}{2}, \frac{n}{2}\right), n \geq 2, \nu \in[0,1], \gamma=\alpha+2 \nu$.

Proof. We have the identity with $v(t)=U(-t) u(t)$ and $w(t, x)=\left(e^{\frac{i|x|^{2}}{2 t}}-1\right) v(t, x)$

$$
\begin{aligned}
u(t) & =U(t) v(t)=M(t) D(t) \mathcal{F} v(t)+M(t) D(t) \mathcal{F}(M(t)-1) v(t) \\
& =\frac{e^{\frac{i|x|^{2}}{2 t}}}{(2 \pi i t)^{\frac{n}{2}}} \int e^{-i y \frac{x}{t}} v(t, y)\left\{1+\left(e^{\frac{i|y|^{2}}{2 t}}-1\right)\right\} d y \\
& =\frac{e^{\frac{i|x|^{2}}{2 t}}}{(i t)^{\frac{n}{2}}}\left(\hat{v}\left(t, \frac{x}{t}\right)+\hat{w}\left(t, \frac{x}{t}\right)\right) .
\end{aligned}
$$

We get the estimate

$$
\left|e^{\frac{i|y|^{2}}{2 t}}-1\right|=2\left|\sin \frac{|y|^{2}}{4 t}\right| \leq \min \left(2, \frac{|y|^{2}}{2|t|}\right) \leq 2^{1-2 \nu} \frac{|y|^{2 \nu}}{|t|^{\nu}}
$$

for any $\nu$ satisfying $0 \leq \nu \leq 1$, and by a direct calculation we see that

$$
\left\|f\left(\frac{\dot{-}}{t}\right)\right\|_{p}=|t|^{n / p}\|f\|_{p}
$$

Applying equality (2.4) and estimate (2.3) to identity (2.2) and using Lemma 2.1 with $p=p(\alpha), a=1, r=2, j=0, m=\alpha$, we get

$$
\begin{aligned}
\|u(t)\|_{p} & \leq C|t|^{-\alpha}\left(\|\hat{v}(t)\|_{p}+\|\hat{w}(t)\|_{p}\right) \leq C|t|^{-\alpha}\left(\|\hat{v}(t)\|_{p}+\|\hat{w}(t)\|_{\alpha, 0}\right) \\
& \leq C|t|^{-\alpha}\left(\|\hat{v}(t)\|_{p}+\|w(t)\|_{0, \alpha}\right) \leq C|t|^{-\alpha}\left(\|\hat{v}(t)\|_{p}+|t|^{-\nu}\|v(t)\|_{0, \gamma}\right) .
\end{aligned}
$$

This implies the lemma. $\quad$ ]

Lemma 2.3. We let $u(t, x)$ be a smooth function and $0<\gamma<\frac{n}{2}$. Then the following estimates

$$
\left|\operatorname{Im}\left(|x|^{\gamma} U(-t) f\left(|u|^{2}\right) u(t),|x|^{\gamma} U(-t) u(t)\right)\right| \leq C\|u\|_{2 n /(n-1)}^{2}\|U(-t) u\|_{\dot{H}^{0, \gamma}}^{2}
$$

and

$$
\left|\operatorname{Im}\left((-\Delta)^{\gamma / 2} f\left(|u|^{2}\right) u(t),(-\Delta)^{\gamma / 2} U(-t) u(t)\right)\right| \leq C\|u\|_{2 n /(n-1)}^{2}\|u\|_{\dot{H}^{\gamma, 0}}^{2}
$$


are valid.

Proof. Let us consider only the case $0<\gamma<1$ since the other cases are treated analogously. By the relation $M(t)\left(-t^{2} \Delta\right)^{\gamma / 2} M(-t)=U(t)|x|^{\gamma} U(-t)$ we have with $g=M(-t) u$ and $f=f\left(|u|^{2}\right)=f\left(|g|^{2}\right)$

$$
\begin{aligned}
& \left|\operatorname{Im}\left(|x|^{\gamma} U(-t) f\left(|u|^{2}\right) u,|x|^{\gamma} U(-t) u\right)\right|=\left|\operatorname{Im}\left(\left(-t^{2} \Delta\right)^{\gamma / 2} f g,\left(-t^{2} \Delta\right)^{\gamma / 2} g\right)\right| \\
& =\left|\operatorname{Im}\left(\left(-t^{2} \Delta\right)^{\gamma / 2} f g-f\left(-t^{2} \Delta\right)^{\gamma / 2} g,\left(-t^{2} \Delta\right)^{\gamma / 2} g\right)\right| \\
& \leq C\|g\|_{2 n /(n-2 \gamma)}\left\|\left(-t^{2} \Delta\right)^{\gamma / 2} f\right\|_{n / \gamma}\left\|\left(-t^{2} \Delta\right)^{\gamma / 2} g\right\|
\end{aligned}
$$

where we have used the fractional Leibniz rule which is proved in [15]. Since $f=$ $V *|u|^{2}=C(-\Delta)^{-(n-1) / 2}|g|^{2}$ with a certain constant $C$ (see [16]) we obtain by Lemma 2.1 with $j=0, m=n-1-\gamma, p=\frac{n}{\gamma}, r=\frac{n}{n-1}, a=1$

$$
\begin{aligned}
& \left|\operatorname{Im}\left(|x|^{\gamma} U(-t)\left(V *|u|^{2}\right) u,|x|^{\gamma} U(-t) u\right)\right| \\
& \leq C|t|^{\gamma}\|g\|_{2 n /(n-2 \gamma)}\left\|(-\Delta)^{-(n-1-\gamma) / 2}|g|^{2}\right\|_{n / \gamma}\left\||x|^{\gamma} U(-t) u\right\| \\
& \leq C|t|^{\gamma}\|g\|_{2 n /(n-2 \gamma)}\|u\|_{2 n /(n-1)}^{2}\left\||x|^{\gamma} U(-t) u\right\| \\
& \leq C\|u\|_{2 n /(n-1)}^{2}\|U(-t) u\|_{\dot{H}^{0, \gamma}}^{2} .
\end{aligned}
$$

The second estimate of the lemma follows from the same argument as in the proof of the first one, and so we omit it. [

\section{$\S 3$ Proofs of Theorems 1.1-1.2.}

To clarify the idea of the proof of the Theorems we only show a priori estimates of local solutions to (NLS). For that purpose we assume that the following local existence theorem holds.

Theorem 3.1. We assume that $\left\|u_{0}\right\|_{\gamma, 0}+\left\|u_{0}\right\|_{0, \gamma}=\epsilon \leq \epsilon^{\prime}$ and $\epsilon$ is sufficiently small, where $\frac{1}{2}<\gamma<\frac{n}{2}$. Then there exists a finite time interval $[-T, T]$ with $T>1$ and a unique solution $u$ of (1.1) such that

$$
\|u\|_{X_{T}} \leq C \epsilon^{\prime}
$$

For the proof of Theorem 3.1, see,e.g, [1],[7],[13],[15]. 
Lemma 3.1. Let $u$ be the local solutions to (1.1) stated in Theorem 3.1. Then we have

$$
\sup _{t \in[-T, T]}(1+|t|)^{-C \epsilon^{\prime}}\left(\|u(t)\|_{\gamma, 0}+\|U(-t) u(t)\|_{0, \gamma}\right) \leq C \epsilon
$$

Proof. By using the commutation relation $\left[L,|J|^{\gamma}\right]=0$, where $L=i \partial_{t}+\frac{1}{2} \Delta$ we get from the Hartree equation (1.1)

$$
L|J|^{\gamma} u=|J|^{\gamma} f\left(|u|^{2}\right) u
$$

Multiplying both sides of this equation by $\overline{|J|^{\gamma} u}$ and using Lemma 2.3, we obtain

$$
\left\||J|^{\gamma} u(t)\right\|^{2} \leq\left\||x|^{\gamma} u_{0}\right\|^{2}+C \int_{0}^{t}\|u(s)\|_{2 n /(n-1)}^{2}\left\||J|^{\gamma} u(s)\right\|^{2} d s .
$$

We use the estimate $\|u(t)\|_{p\left(\frac{1}{2}\right)} \leq C \epsilon(1+|t|)^{-\frac{1}{2}}$ of Theorem 3.1 to get

$$
\left\||J|^{\gamma} u(t)\right\|^{2} \leq\left\||x|^{\gamma} u_{0}\right\|^{2}+C \epsilon^{\prime} \int_{0}^{t}(1+s)^{-1}\left\||J|^{\gamma} u(s)\right\|^{2} d s
$$

whence via the Gronwall's inequality we find the estimate $\left\||J|^{\gamma} u(t)\right\| \leq\left\||x|^{\gamma} u_{0}\right\|(1+$ t) ${ }^{C \epsilon^{\prime}}$ which implies

$$
(1+t)^{-C \epsilon^{\prime}}\left\||x|^{\gamma} U(-t) u(t)\right\| \leq\left\||x|^{\gamma} u_{0}\right\|
$$

In the same way as in the proof of (3.1) we have

$$
(1+t)^{-C \epsilon^{\prime}}\|u(t)\|_{\gamma, 0} \leq C\left\|u_{0}\right\|_{\gamma, 0}
$$

The lemma follows from (3.1) and (3.2).

Lemma 3.2. Let $u$ be the local solutions to (1.1) stated in Theorem 3.1. Then we have

$$
\sup _{\alpha \in\left[\frac{1}{2}, \sigma\right]} \sup _{t \in[-T, T]}(1+|t|)^{\alpha}\|u(t)\|_{p(\alpha)} \leq C \epsilon,
$$

where $\frac{1}{2}<\sigma<\gamma, p(\alpha)=\frac{2 n}{n-2 \alpha}$.

Proof. By Lemma 3.1 and Theorem 3.1 we have

$$
\sup _{\alpha \in\left[\frac{1}{2}, \sigma\right]} \sup _{t \in[-1,1]}(1+|t|)^{\alpha}\|u(t)\|_{p(\alpha)} \leq C \epsilon .
$$


We assume that $t \geq 1$. From Lemma 2.2 and Lemma 3.1 it follows that

$$
\|u(t)\|_{p(\alpha)} \leq C \epsilon t^{-\alpha-\beta+C \epsilon}+C t^{-\alpha}\|\mathcal{F} U(-t) u(t)\|_{p(\alpha)}
$$

for any $\alpha \in\left[\frac{1}{2}, \sigma\right]$, where $\beta=\min \left(1, \frac{\gamma-\sigma}{2}\right)$. Multiplying both sides of $(1.1)$ by $U(-t)$ we obtain

$$
i(U(-t) u(t))_{t}+U(-t) f\left(|u|^{2}\right) u=0
$$

whence in view of identity (1.4) we have

$$
\left.i \hat{v}_{t}-t^{-1} f\left(|\hat{v}|^{2}\right) \hat{v}=t^{-1}\left(I_{1}(t)+I_{2}(t)\right)\right)
$$

where

$$
\begin{gathered}
I_{1}(t)=\mathcal{F}(M(-t)-1) \mathcal{F}^{-1} f\left(|\mathcal{F} M(t) v|^{2}\right) \mathcal{F} M(t) v \\
I_{2}(t)=f\left(|\mathcal{F} M(t) v|^{2}\right) \mathcal{F} M(t) v-f\left(|\hat{v}|^{2}\right) \hat{v}
\end{gathered}
$$

Then by (3.5)

$$
i \hat{w}_{t}=B(t)\left(t^{-1}\left\{I_{1}(t)+I_{2}(t)\right\}\right)
$$

with $\hat{v} B(t)=\hat{w}$ and $B(t)=\exp \left(i \int_{1}^{t} f\left(|\hat{v}|^{2}\right) \frac{d \tau}{\tau}\right)$. Integrating (3.6) with respect to $t$ from 1 to $t$, we get

$$
\hat{w}(t)=\hat{w}(1)+i \int_{1}^{t} B(\tau)\left(\left\{I_{1}(\tau)+I_{2}(\tau)\right\}\right) \frac{d \tau}{\tau} .
$$

In the same way as in the proof of Lemma 2.3 we have with $\frac{1}{2}<\theta \leq \gamma$ (We let now $\gamma<1$, since the case $1 \leq \gamma<\frac{n}{2}$ is treated analogously.)

$$
\begin{aligned}
\| f\left(h_{1} h_{2}\right) & h_{3} \|_{\theta, 0} \leq C\left(\|f\|_{\infty}\left\|h_{3}\right\|+\left\|(-\Delta)^{\theta / 2}\left(f h_{3}\right)\right\|\right) \\
& \leq C\left(\|f\|_{\infty}\left\|h_{3}\right\|+\left\|(-\Delta)^{\theta / 2}\left(f h_{3}\right)-f(-\Delta)^{\theta / 2} h_{3}\right\|+\left\|f(-\Delta)^{\theta / 2} h_{3}\right\|\right) \\
& \leq C\left(\|f\|_{\infty}\left\|h_{3}\right\|_{\theta, 0}+\left\|h_{3}\right\|_{p(\theta)}\left\|(-\Delta)^{\theta / 2} f\right\|_{n / \theta}\right) \\
& \leq C\left(\|f\|_{\infty}\left\|h_{3}\right\|_{\theta, 0}+\left\|h_{3}\right\|_{p(\theta)}\left\|(-\Delta)^{-(n-1-\theta) / 2} h_{1} h_{2}\right\|_{n / \theta}\right) \\
& \leq C\left\|h_{3}\right\|_{\theta, 0}\left(\|f\|_{\infty}+\left\|h_{1}\right\|_{p\left(\frac{1}{2}\right)}\left\|h_{2}\right\|_{p\left(\frac{1}{2}\right)}\right) \\
& \leq C\left\|h_{3}\right\|_{\theta, 0}\left(\left\|h_{1}\right\|_{q}\left\|h_{2}\right\|_{q}+\left\|h_{1}\right\|_{r}\left\|h_{2}\right\|_{r}+\left\|h_{1}\right\|_{\frac{1}{2}, 0}\|\| h_{2} \|_{\frac{1}{2}, 0}\right) \leq C \prod_{j=1}^{3}\left\|h_{j}\right\|_{\theta, 0}
\end{aligned}
$$

where $q<p\left(\frac{1}{2}\right)<r<p(\theta)$. We use (3.8) and (2.3) to obtain with $h=M(t) v$ and $0 \leq \theta \leq \sigma$

$$
\begin{aligned}
\left\|I_{1}(t)\right\|_{\theta, 0} & \leq C\left\|\mathcal{F}(M(-t)-1) \mathcal{F}^{-1} f\left(|\hat{h}|^{2}\right) \hat{h}\right\|_{\theta, 0} \leq C t^{-\omega}\left\|\mathcal{F}^{-1} f\left(|\hat{h}|^{2}\right) \hat{h}\right\|_{0, \gamma} \\
& \leq C t^{-\omega}\left\|f\left(|\hat{h}|^{2}\right) \hat{h}\right\|_{\gamma, 0} \leq C t^{-\omega}\|\hat{h}\|_{\gamma, 0}^{3} \leq C t^{-\omega}\|v\|_{0, \gamma}^{3}
\end{aligned}
$$


where $\omega=\min \left(1, \frac{\gamma-\theta}{2}\right)$. We easily see that

$$
f\left(|\hat{h}|^{2}\right) \hat{h}-f\left(|\hat{v}|^{2}\right) \hat{v}=f\left(|\hat{h}|^{2}\right)(\hat{h}-\hat{v})+f((\hat{h}-\hat{v}) \overline{\hat{h}}) \hat{v}+f((\overline{\hat{h}}-\overline{\hat{v}}) \hat{v}) \hat{v}
$$

Applying (3.8) to (3.10), using (2.3) and Lemma 2.1, we obtain

$$
\begin{aligned}
\left\|I_{2}(t)\right\|_{\sigma, 0} & =\left\|f\left(|\hat{h}|^{2}\right) \hat{h}-f\left(|\hat{v}|^{2}\right) \hat{v}\right\|_{\sigma, 0} \leq C\|\hat{h}-\hat{v}\|_{\sigma, 0}\left(\|\hat{h}\|_{\sigma, 0}^{2}+\|\hat{v}\|_{\sigma, 0}^{2}\right) \\
& \leq C\|(M(t)-1) v\|_{0, \sigma}\|v\|_{0, \sigma}^{2} \leq C t^{-\beta}\|v\|_{0, \gamma}^{3}
\end{aligned}
$$

where $\frac{1}{2}<\sigma<\gamma<\frac{n}{2}, \beta=\min \left(1, \frac{\gamma-\sigma}{2}\right)$. By (3.7), (3.9), (3.11) and Lemma 3.1 we have

$$
\begin{gathered}
\|\mathcal{F} U(-t) u\|_{p(\alpha)}=\|\hat{v}\|_{p(\alpha)}=\|\hat{w}\|_{p(\alpha)} \leq C \epsilon+C \int_{1}^{t}\left(\left\|I_{1}(\tau)\right\|_{p(\alpha)}+\left\|I_{2}(\tau)\right\|_{p(\alpha)}\right) \frac{d \tau}{\tau} \\
\leq C \epsilon+C \int_{1}^{t}\left(\left\|I_{1}(\tau)\right\|_{\alpha, 0}+\left\|I_{2}(\tau)\right\|_{\alpha, 0}\right) \frac{d \tau}{\tau} \leq C \epsilon \int_{1}^{t} \tau^{-1-\beta+C \epsilon} d \tau \leq C \epsilon
\end{gathered}
$$

We apply (3.12) to (3.4) to get the lemma. $\quad$,

In the same way as in the proof of Lemma 3.1 we have by Theorem 3.1 and Lemma 3.2

Lemma 3.3. Let $u$ be the local solutions to (1.1) stated in Theorem 3.1. Then we have

$$
\sup _{t \in[-T, T]}(1+|t|)^{-C \epsilon}\left(\|u(t)\|_{\gamma, 0}+\|U(-t) u(t)\|_{0, \gamma}\right) \leq C \epsilon .
$$

We are now in a position to prove Theorems 1.1-1.2.

Proof of Theorem 1.1. We have by Lemma 3.2 and Lemma 3.3

$$
\||u|\| X_{T} \leq C\left(\left\|u_{0}\right\|_{\gamma, 0}+\left\|u_{0}\right\|_{0, \gamma}\right)=C \epsilon .
$$

We take $\epsilon$ satisfying $C \epsilon \leq \epsilon^{\prime}$. Then the standard continuation argument yields the result because our constant $C$ does not depend on the existence time $T$. $\quad$

Proof of Theorem 1.2. By (3.7), (3.9) and (3.11) and Theorem 1.1 we have

$$
\|\hat{w}(t)-\hat{w}(s)\|_{\sigma, 0} \leq C \epsilon s^{-\beta+C \epsilon}
$$


for $t>s \geq 1$, where $\frac{1}{2}<\sigma<\gamma, \beta=\min \left(1, \frac{\gamma-\sigma}{2}\right)$. Therefore we find that there exists a unique function $\hat{w}_{+} \in H^{\sigma, 0}$ such that

$$
\left\|\hat{w}(t)-\hat{w}_{+}\right\|_{\sigma, 0} \leq C \epsilon t^{-\beta+C \epsilon} .
$$

In the same way as in the proof of (3.9) and (3.11) we have

$$
\left\|I_{1}(t)\right\|_{0, \sigma}+\left\|I_{2}(t)\right\|_{0, \sigma} \leq C t^{-\beta+C \epsilon}\left(\|v\|_{0, \gamma}+\|v\|_{\gamma, 0}\right)^{3} \leq C \epsilon t^{-\beta+C \epsilon} .
$$

Hence from (3.7) we see that $\hat{w}_{+} \in H^{0, \sigma}$ and

$$
\left\|\hat{w}(t)-\hat{w}_{+}\right\|_{0, \sigma} \leq C \epsilon t^{-\beta+C \epsilon} .
$$

By Lemma 2.1 with $p=2 n, j=0, a=1, m=n-1, r=\frac{2 n}{2 n-1}$ we find that

$$
\left\|f\left(h_{1} h_{2}\right)\right\|_{2 n}=C\left\|(-\Delta)^{-(n-1) / 2}\left(h_{1} h_{2}\right)\right\|_{2 n} \leq C\left\|h_{1}\right\|\left\|h_{2}\right\|_{p\left(\frac{1}{2}\right)} \leq C\left\|h_{1}\right\|\left\|h_{2}\right\|_{\frac{1}{2}, 0} .
$$

Let us consider now the case $\frac{1}{2}<\sigma<1$. The other cases can be treated analoguously. We use estimate (3.17) to get the following estimate with $\frac{1}{2}<\sigma<\gamma$ (Let us consider now the case $\frac{1}{2}<\gamma<1$, since the case $1 \leq \gamma<\frac{n}{2}$ is treated analogously.)

$$
\begin{aligned}
& \left\|f\left(h_{1} h_{2}\right)\right\|_{\dot{B}_{2 n, 2}^{\sigma}}=\left(\int_{0}^{\infty} y^{-1-2 \sigma} \sup _{|z| \leq y}\left\|\left(f_{(z)}-f\right)\right\|_{2 n}^{2} d y\right)^{1 / 2} \\
& \leq C\left\|h_{2}\right\|_{\frac{1}{2}, 0}\left(\int_{0}^{\infty} y^{-1-2 \sigma} \sup _{|z| \leq y}\left\|\left(h_{1(z)}-h_{1}\right)\right\|^{2} d y\right)^{1 / 2} \\
& \quad+C\left\|h_{1}\right\|_{\frac{1}{2}, 0}\left(\int_{0}^{\infty} y^{-1-2 \sigma} \sup _{|z| \leq y}\left\|\left(h_{2(z)}-h_{2}\right)\right\|^{2} d y\right)^{1 / 2} \leq C\left\|h_{1}\right\|_{\sigma, 0}\left\|h_{2}\right\|_{\sigma, 0}
\end{aligned}
$$

whence we obtain

$$
\begin{gathered}
\left.\left\|f\left(|\hat{w}(t)|^{2}\right)-f\left(|\hat{w}(s)|^{2}\right)\right\|_{\dot{B}_{2 n, 2}^{\sigma}}=\| f((\hat{w}(t)-\hat{w}(s)) \overline{\hat{w}(t)})+f(\overline{\hat{w}(t)}-\overline{\hat{w}(s)}) \hat{w}(t)\right) \|_{\dot{B}_{2 n, 2}^{\sigma}} \\
\leq C\|\hat{w}(t)-\hat{w}(s)\|_{\sigma, 0}\left(\|\hat{w}(t)\|_{\sigma, 0}+\|\hat{w}(s)\|_{\sigma, 0}\right) \leq C s^{-\beta+C \epsilon}\left(\left\|u_{0}\right\|_{0, \gamma}+\left\|u_{0}\right\|_{\gamma, 0}\right)
\end{gathered}
$$

for all $1<s<t$, where $\beta=\min \left(1, \frac{\gamma-\sigma}{2}\right)$.

We now let $\Psi(t)=\int_{1}^{t}\left(f\left(|\hat{w}(\tau)|^{2}\right)-f\left(|\hat{w}(t)|^{2}\right)\right) \frac{d \tau}{\tau}$. Then

$$
\Psi(t)-\Psi(s)=\int_{s}^{t}\left(f\left(|\hat{w}(\tau)|^{2}\right)-f\left(|\hat{w}(t)|^{2}\right)\right) \frac{d \tau}{\tau}-\left(f\left(|\hat{w}(t)|^{2}\right)-f\left(|\hat{w}(s)|^{2}\right)\right) \log s
$$


where $1<s<\tau<t$. We apply (3.19) to (3.20) to get for all $1<s<t$

$$
\|\Psi(t)-\Psi(s)\|_{\dot{B}_{2 n, 2}^{\sigma}} \leq C \epsilon s^{-\beta+C \epsilon} .
$$

This implies that there exists a unique real valued function $\Phi \in \dot{B}_{2 n, 2}^{\sigma}$ such that $\lim _{t \rightarrow \infty} \Psi(t)=\Phi \quad$ in $\quad \dot{B}_{2 n, 2}^{\sigma}$. We let $t \rightarrow \infty$ in (3.21). Then

$$
\|\Phi-\Psi(t)\|_{\dot{B}_{2 n, 2}^{\sigma}} \leq C \epsilon t^{-\beta+C \epsilon} .
$$

We denote $\hat{u}_{+}=\hat{w}_{+} \exp (-i \Phi)$. By the facts that $\Phi \in \dot{B}_{2 n, 2}^{\sigma}, \hat{w}_{+} \in H^{\sigma, 0} \cap H^{0, \sigma}$ and Lemma 2.1 we see that $\hat{u}_{+} \in H^{\sigma, 0} \cap H^{0, \sigma}$ and we also have by Theorem 1.1, (3.14), (3.21) and (3.22)

$$
\begin{aligned}
\left\|u_{+}\right\|_{0, \sigma} & =\left\|\hat{u}_{+}\right\|_{\sigma, 0}=\left\|\exp (i \Phi) \hat{w}_{+}\right\|_{\sigma, 0} \leq C\left(\left\|w_{+}\right\|+\left\|\hat{w}_{+} \exp (i \Phi)\right\|_{\dot{B}_{2,2}^{\sigma}}\right) \\
& \leq C \epsilon+C\left(\int_{0}^{\infty} y^{-1-2 \sigma} \sup _{|z| \leq y}\left\|\left(\hat{w}_{+(z)}-\hat{w}_{+}\right) \exp \left(i \Phi_{(z)}\right)\right\|^{2} d y\right)^{1 / 2} \\
& +C\left\|\hat{w}_{+}\right\|_{p\left(\frac{1}{2}\right)}\left(\int_{0}^{\infty} y^{-1-2 \sigma} \sup _{|z| \leq y}\left\|\Phi_{(z)}-\Phi\right\|_{2 n}^{2} d y\right)^{1 / 2} \\
& \leq C \epsilon+C\left\|\hat{w}_{+}\right\|_{\sigma, 0}+C\left\|\hat{w}_{+}\right\|_{\frac{1}{2}, 0}\|\Phi\|_{\dot{B}_{2 n, 2}^{\sigma}} \leq C \epsilon .
\end{aligned}
$$

We easily find that the following identity holds

$$
\int_{1}^{t} f\left(|\hat{w}(\tau)|^{2}\right) \frac{d \tau}{\tau}=f\left(\left|\hat{w}_{+}\right|^{2}\right) \log t+\Phi+(\Psi(t)-\Phi)+\left(f\left(|\hat{w}(t)|^{2}\right)-f\left(\left|\hat{w}_{+}\right|^{2}\right)\right) \log t
$$

In the same way as in the proof of (3.11), (3.17) we have

$$
\begin{aligned}
\left\|I_{2}(t)\right\| & =\left\|f\left(|\hat{h}|^{2}\right) \hat{h}-f\left(|\hat{v}|^{2}\right) \hat{v}\right\| \leq\|\hat{h}-\hat{v}\|\left\|f\left(|\hat{h}|^{2}\right)\right\|_{\infty} \\
& +\|\hat{v}\|_{p\left(\frac{1}{2}\right)}\left(\|f((\hat{h}-\hat{v}) \overline{\hat{h}})\|_{2 n}+\|f((\overline{\hat{h}}-\overline{\hat{v}}) \hat{v})\|_{2 n}\right) \leq C\|\hat{h}-\hat{v}\|\left(\|\hat{h}\|_{\sigma, 0}^{2}+\|\hat{v}\|_{\sigma, 0}^{2}\right) \\
& \leq C\|(M(t)-1) v\|\|v\|_{0, \sigma}^{2} \leq C t^{-\mu}\|v\|_{0, \gamma}^{3} \leq C t^{-\mu+C \epsilon}\left(\|v\|_{0, \gamma}+\|v\|_{\gamma, 0}\right)^{3} \\
& \leq C \epsilon t^{-\mu+C \epsilon}
\end{aligned}
$$

where $\mu=\min \left(1, \frac{\gamma}{2}\right)$. Hence by virtue of (3.9) with $\theta=0$ and (3.25) we see from (3.7) that

$$
\left\|\hat{w}(t)-\hat{w}_{+}\right\| \leq C \epsilon t^{-\mu+C \epsilon} .
$$

Using (3.17) and (3.26), analogouosly to (3.19) we get

$$
\begin{aligned}
& \left.\left\|f\left(|\hat{w}(t)|^{2}\right)-f\left(|\hat{w}(s)|^{2}\right)\right\|_{2 n}=\| f((\hat{w}(t)-\hat{w}(s)) \overline{\hat{w}(t)})+f(\overline{\hat{w}(t)}-\overline{\hat{w}(s)}) \hat{w}(t)\right) \|_{2 n} \\
& \quad \leq C\|\hat{w}(t)-\hat{w}(s)\|\left(\|\hat{w}(t)\|_{p\left(\frac{1}{2}\right)}+\|\hat{w}(s)\|_{p\left(\frac{1}{2}\right)}\right) \leq C s^{-\mu+C \epsilon}\left(\left\|u_{0}\right\|_{0, \gamma}+\left\|u_{0}\right\|_{\gamma, 0}\right)
\end{aligned}
$$


and therefore we obtain

$$
\|\Phi-\Psi(t)\|_{2 n} \leq C \epsilon t^{-\mu+C \epsilon}
$$

By (3.24), (3.27) and (3.28) we have

$$
\left\|\int_{1}^{t} f\left(|\hat{w}(\tau)|^{2}\right) \frac{d \tau}{\tau}-f\left(\left|\hat{w}_{+}\right|^{2}\right) \log t-\Phi\right\|_{2 n} \leq C \epsilon t^{-\mu+C \epsilon} .
$$

Since $\hat{w}(t)=B(t) \mathcal{F} U(-t) u(t)=\exp \left(i \int_{1}^{t} f\left(|\hat{w}(\tau)|^{2}\right) \frac{d \tau}{\tau}\right) \mathcal{F} U(-t) u(t)$, we have in view of $(3.14),(3.26)$ and $(3.29)$

$$
\begin{aligned}
\| \mathcal{F} U(-t) u(t) & -\hat{u}_{+} \exp \left(-i f\left(\left|\hat{u}_{+}\right|^{2}\right) \log t\right) \| \\
& =\left\|\hat{w}(t) \exp \left(-i \int_{1}^{t} f\left(|\hat{w}(\tau)|^{2}\right) \frac{d \tau}{\tau}\right)-\hat{u}_{+} \exp \left(-i f\left(\left|\hat{u}_{+}\right|^{2}\right) \log t\right)\right\| \\
& \left.\leq\left\|\hat{w}(t)-\hat{w}_{+}\right\|+C \| \hat{w}_{+}\left(\int_{1}^{t} f\left(|\hat{w}(\tau)|^{2}\right) \frac{d \tau}{\tau}\right)-f\left(\left|\hat{u}_{+}\right|^{2}\right) \log t-\Phi\right) \| \\
& \leq C \epsilon t^{-\mu+C \epsilon}+C\left\|\hat{w}_{+}\right\|_{p\left(\frac{1}{2}\right)}\left\|\int_{1}^{t} f\left(|\hat{w}(\tau)|^{2}\right) \frac{d \tau}{\tau}-f\left(\left|\hat{w}_{+}\right|^{2}\right) \log t-\Phi\right\|_{2 n} \\
& \leq C \epsilon t^{-\mu+C \epsilon} .
\end{aligned}
$$

By (2.3) and Theorem 1.1

$$
\begin{aligned}
\| u(t) & -M(t) D(t) \mathcal{F} U(-t) u(t)\|=\| M(t) D(t) \mathcal{F}(M(t)-1) U(-t) u(t) \| \\
& =\|(M(t)-1) U(-t) u(t)\| \leq C t^{-\mu}\|U(-t) u(t)\|_{0, \gamma} \leq C \epsilon t^{-\mu+C \epsilon}
\end{aligned}
$$

Via (3.23), (3.30) and (3.31) it follows that

$$
\begin{aligned}
\| u(t) & -\exp \left(-i\left(f\left(\left|\hat{u}_{+}\right|^{2}\right)\left(\frac{x}{t}\right) \log t\right) U(t) u_{+} \|\right. \\
& =\left\|u(t)-M(t) D(t) \exp \left(-i f\left(\left|\hat{u}_{+}\right|^{2}\right) \log t\right) \mathcal{F} M(t) u_{+}\right\| \\
& \leq\|u(t)-M(t) D(t) \mathcal{F} U(-t) u(t)\| \\
& +\left\|M(t) D(t)\left(\mathcal{F} U(-t) u(t)-\hat{u}_{+} \exp \left(-i f\left(\left|\hat{u}_{+}\right|^{2}\right) \log t\right)\right)\right\| \\
& +\left\|M(t) D(t) \exp \left(-i f\left(\left|\hat{u}_{+}\right|^{2}\right) \log t\right) \mathcal{F}(M(t)-1) u_{+}\right\| \\
& \leq C \epsilon t^{-\mu+C \epsilon}+C t^{-\mu+C \epsilon}\left\|u_{+}\right\|_{0, \sigma} \leq C \epsilon t^{-\mu+C \epsilon},
\end{aligned}
$$

where we chose the value $\sigma \in\left(\frac{1}{2}, \gamma\right)$ to be sufficiently close to $\gamma$. From (3.32) Theorem 1.2 follows.

Acknowledgements. One of the authors (P.I.N.) wish to express his deep gratitude to Consejo Nacional de Ciencia y Tecnologia de Mexico (Conacyt) for the support. He is also grateful to Instituto de Fisica y Matematicas de Universidad Michoacana for kind hospitality. 


\section{REFERENCES}

1. J.Bergh and J. Löfström, Interpoolation spaces, Berlin, Heidelberg, New York, Springer, 1976.

2. J.P.Chadam and R.T.Glassey, Global existence of solutions to the Cauchy problem for time dependent Schrödinger Hartree equations, J. Math. Phys. 16 (1975), 12-1130.

3. J.P.Dias and M.Figueira, Conservation laws and time decay for the solutions of some nonlinear Schrödinger-Hartree equations and systems, J.Math. Anal. Appl. 84 (1981), 486-508.

4. A.Friedman, Partial Differential Equations, New York, Holt-Rinehart and Winston, 1969.

5. J.Ginibre and T.Ozawa, Long range scattering for nonlinear Schrödinger and Hartree equations in space dimension $n \geq 2$, Commun. Math. Phys. 151 (1993), 619-645.

6. R.T.Glassey, Asymptotic behavior of solutions to certain nonlinear Schrödinger-Hartree equations, Commun. Math. Phys. 53 (1977), 9-18.

7. J.Ginibre and G.Velo, On a class of nonlinear Schrödinger equations with non-local interactions, Math. Z. 170 (1980), 109-136.

8. N.Hayashi, Asymptotic behavior of solutions to time-dependent Hartree equations, Nonlinear Anal. TMA 12 (1988), 313-319.

9. N.Hayashi and P.I.Naumkin, Asymptotics for large time behavior of solutions to the nonlinear Schrödinger and Hartree equations, preprint (1996).

10. N.Hayashi and T.Ozawa, Time decay of solutions to the Cauchy problem for time-dependent Schrödinger-Hartree equations, Commun. Math. Phys, 110 (1987), 467-478.

11. N.Hayashi and T.Ozawa, Scattering theory in the weighted $L^{2}\left(\mathbf{R}^{n}\right)$ spaces for some Schrödinger equations, Ann. IHP (Phys. Théor.) 48 (1988), 17-37.

12. N.Hayashi and Y.Tsutsumi, Scattering theory for Hartree type equations, Ann. IHP (Phys. Théor.) 46 (1987), 187-213.

13. H.Hirata, The Cauchy problem for Hartree type Schrödinger equation in weighted Sobolev space, J. Fac. Sci. Univ. Tokyo, Sect. IA, Math 38 (1991), 567-588.

14. H.Hirata, Large time behavior of solutions for Hartree equation with long range interaction, Tokyo J. Math. 18 (1995), 167-177.

15. C.E.Kenig, G.Ponce and L.Vega, Well-posedness and scattering results for the generalized Korteweg-de Vries equation via contraction principle, Comm. Pure Appl. Math. 46 (1993), $527-620$.

16. E.M.Stein, Singular Integral and Differentiability Properties of Functions, Princeton Univ. Press, Princeton Math. Series 30. 